\title{
The impact of solutions for chronic disease prevention and management
}

\author{
Mark Donnelly • Chris Nugent • Cristiano Paggetti
}

Received: 20 March 2013 / Accepted: 21 March 2013 /Published online: 9 April 2013

(C) IUPESM and Springer-Verlag Berlin Heidelberg 2013

We are now living in an era where lower-cost, higherperformance technology has the potential to become truly ubiquitous as it integrates seamlessly within our everyday lives. It could be argued that the realization of these concepts will be most significant towards promoting independent living and increasing the quality of life for the rising numbers of older people and with it, the prevalence of chronic conditions such as cardiovascular disease, cancer and dementia. Such illnesses are placing significant and unsustainable pressure upon health and social care services across the globe. In an effort to help to alleviate some of these pressures, efforts are being made to investigate the potential to embed assistive technology within home environments and beyond. Indeed, studies have shown that older people and those with chronic illnesses often prefer to remain living within their own homes for as long as possible. A challenge in supporting their independent living is to ensure that technology driven health and social care solutions provide an equal quality of care when compared to institutional care facilities. Key to this success is the provision of 'smart' homes, which are able to provide automated and personalized levels of support, based on the profiles of each occupant.

Realizing a smart home is, however, not a straightforward task. They are typically equipped with a large number of sensors, which are attached to household objects or embedded

\section{Donnelly $(\bowtie) \cdot$ C. Nugent}

School of Computing and Mathematics,

University of Ulster at Jordanstown, Shore Road,

Newtownabbey, Co. Antrim BT37 0QB, UK

e-mail: mp.donnelly@ulster.ac.uk

C. Nugent

e-mail: cd.nugent@ulster.ac.uk

C. Paggetti

I + S.r.1., Piazza Puccini 26,

50144 Firenze, Italy

e-mail: c.paggetti@i-piu.it within appliances hence supporting the ability to track occupant's locations and their interaction with appliances. These sensors generate a large amount of data, which must then be organized and mined using computation intelligence approaches to extract features that contribute most to the recognition of particular behaviors, environmental changes or to identifying activities of daily living. A large body of research in this area now exists; however, we have not yet witnessed the advent of smart homes on a large scale of deployment. What is needed is a coordinated and collaborative approach to technology development based on a user-centered design methodology with strong industrial and health and social care services support to ensure the large-scale uptake, which is currently lacking.

The objectives of this Special Issue were firstly to review the most recent advances in the development of solutions for chronic disease prevention and management from a technology perspective and secondly to consider the impact that such solutions are having on health and social care provision. The consolidation of such an issue aims to provide a collection of work with the opportunity of exchanging new ideas and sharing lessons learnt.

The Theme of the Special Issue follows the theme of the 10th International Conference On Smart homes and health Telematics (ICOST) that was held in Italy in 2012. The focus of the conference was related to considering the impact, which health technologies are currently having at a real deployment level in trying to address the issues surrounding long term chronic illnesses. This Special issue includes extended papers from a number of notable authors whose work was well received by delegates attending ICOST 2012. The overarching aim of the Special Issue was to provide interested readers with an insightful overview into state of the art approaches being applied to provide technology support for long-term chronic illness. Specifically, this Special Issue presents the latest research findings from an international body of authors originating from Spain, France, United Kingdom, Canada, 
Australia and South Korea. The topics addressed can be categorized into: Wearable Computing, Mobile Health, the importance of User Engagement in the design and development of technological solutions aimed towards health, Context-aware computing, Cloud-based Services and, the importance of Formatting and Storage standards.

Solar et al. reiterate the challenges facing current healthcare models due to demographic changes and the associated rise in chronic condition such as Congestive Heart Failure. In an effort to alleviate some of these challenges, they present findings from their recent work involving the design and development of a multi-sensorial body worn platform for supporting multi-parametric remote monitoring in patients diagnosed with CHF.

Leijdekkers and Gay share their experiences in the design, development and commercialization of a suite of mobile health app services, over a 7-year period. Their product, myFitnessCompanion ${ }^{\circledR}$, offers services to support chronic disease management and has been available for consumer download since early 2011. Based on an analysis of data collected from over 5,500 users over a period of 7 months, the authors offer insight into the opportunities of moving beyond research prototypes, outlining their views on the future strategies and challenges for distributing mobile health apps.

Galway et al. leverage several years experience in the design and development of a mobile phone-based video streaming (MPVS) system, aimed for use by people with dementia, to outline an interaction framework that guides stakeholder involvement in the development of assistive technology solutions. This framework may be employed when planning assistive technology development in order to achieve a technology-driven, yet user-led, methodology that leads to informed decision making based on real needs, requirements and feedback.

Allègre et al. introduce an approach to efficiently model user-system interactions within a smart environment allowing for a reduction in the number of physical sensors required to be deployed. They present a context-aware system based on realtime semantic analysis of inhabitant interactions within an environment to infer user location and intention, leading to adapted service delivery.
In a complementary paper, Gouin-Vallerand et al. introduce a micro and macro context awarene model to support the dynamic delivery of services within a smart environment. The micro context awareness revolves around the subjective perception and the understanding an environment entity has of its environment while the macro context awareness is the global, emergent picture that components help build of entities in their environment. Encouragingly, the authors accompany their model with an intelligibility model, which allows for the generation of explanations that rationalize the system's behaviors according to the processed context.

Hussain et al. present their evaluations of a cloud-based Smart Clinical Decision Support System (CDSS) that takes input from diverse modalities such as sensors, user profile information, social media, clinical knowledge base's and medical experts to generate standards based personalized recommendations. This is complemented by an activity recognition framework built upon environmental sensors.

Finally, McDonald et al. highlight the lack of standards that currently exist around the formatting and storage of smart environment sensor data. In their paper, they present an evaluation of a suite of services to support both the formatting and storage of sensor data in a common XML based format and additionally, outline the suite of services they provide for sharing data among researchers.

In summary, this Special Issue of Health and Technology provides the groundings for both new and experienced researchers investigating this domain. It raises many important and yet solved issues from both a technological and user acceptance viewpoint. Indeed, it highlights the key challenges with developing technology to support and manage long-term chronic illnesses. Over the last decade many new solutions have emerged to support the realization of smart homes. Building upon new developments such as those presented in this issue instills confidence that we are making gains in this domain and may someday soon witness truly smart homes.

Mark Donnelly

Chris Nugent

Cristiano Paggetti 